\section{Diurnal intraocular pressure fluctuation and its risk factors in angle-closure and open-angle glaucoma}

${ }^{1}$ Elite School of Optometry, Medical Research Foundation, Chennai, India

2Jadhavbhai Nathamal Singhvi Glaucoma Department, Medical Research Foundation, Sankara Nethralaya,

Chennai, India

${ }^{3}$ Institute of Health and Biomedical Innovation, Queensland University of Technology, Queensland, Australia

${ }^{4}$ VST Glaucoma Centre, Dr Kallam Anji Reddy Campus, LV Prasad Eye Institute, Hyderabad, India

${ }^{5}$ Singapore Eye Research Institute and Singapore National Eye Center, Singapore

Correspondence: L Vijaya, Director, Jadhavbhai Nathamal Singhvi Glaucoma Department, Medical Research Foundation, Sankara Nethralaya, 18/41, College Road, Chennai 600 006, India Tel: +91 4428271616 ; Fax: +91 4428254180 E-mail:drlv@snmail.org

${ }^{6}$ These authors contributed equally to this work.

Received: 10 December 2014

Accepted in revised form: 23 September 2015

Published online:

13 November 2015

\section{Abstract}

Purpose To evaluate intraocular pressure (IOP) fluctuation during office hours and its predictive factors in untreated primary angleclosure suspects (PACS); post-iridotomy primary angle closure (PAC) and primary without IOP-lowering medication(s) as appropriate and medically treated primary open-angle glaucoma (POAG) eyes.

Methods One-hundred seventeen eyes (29 PACS, 30 PAC, 28 PACG, and 30 POAG) of 117 patients were included in this crosssectional study. The subjects underwent hourly IOP measurements with Goldmann tonometer from 0800 to $\mathbf{1 7 0 0}$ hours. Subjects with PAC and PACG had laser peripheral inclusion. SD of office-hour IOP readings was the main outcome measure.

Results IOP fluctuation differed between the groups ( $P=0.01$; Kruskal-Wallis Test). Post hoc Mann-Whitney $U$-tests showed significantly less IOP fluctuation in PACS compared with PACG $(P<0.01)$. Peak office-hour IOP was observed in the morning in untreated subjects and in the early afternoon in treated subjects. A stepwise linear regression model identified the presence of peripheral anterior synechiae (PAS), thickness of lens, large vertical cup-to-disc ratio (VCDR), and PAC category as significant predictive factors associated with office-hour IOP fluctuation.

Conclusions Diurnal IOP fluctuation in asymptomatic PACSs was less than that in treated PACG subjects and was at least comparable to that in treated PAC and POAG thicker the lens, the larger the VCDR, the greater was the IOP fluctuation during office hours.

Eye (2016) 30, 362-368; doi:10.1038/eye.2015.231; published online 13 November 2015 angle-closure glaucoma (PACG) eyes with or iridotomy at least 2 weeks prior to the subjects. The greater the amount of PAS, the
S Srinivasan 1,2,3,6, NS Choudhari ${ }^{2,4,6}$ M Baskaran², ${ }^{2}$ RJ George ${ }^{2}$, B Shantha ${ }^{2}$ and L Vijaya ${ }^{2}$

\section{Introduction}

Intraocular pressure (IOP) is a major risk factor for the development and progression of glaucoma. The amplitude of 24-h IOP fluctuation has been demonstrated to be higher in patients with or suspected to have openangle glaucoma compared with the control group. ${ }^{1,2}$ Fluctuation of IOP, both during ${ }^{3}$ and beyond office hours ${ }^{4}$ has been shown to be an independent risk factor for the progression of open-angle glaucoma. However, available literature on fluctuation of IOP in angle-closure disease is sparse.

Sihota $e t a l^{5}$ reported significantly higher diurnal IOP fluctuation in primary angleclosure glaucoma (PACG) and primary openangle glaucoma (POAG) groups compared with normal controls. However, they did not include angle-closure eyes with $<180^{\circ}$ peripheral anterior synechiae (PAS) on indentation gonioscopy. Diurnal IOP was significantly higher in PACG patients post laser peripheral iridotomy (LPI) and untreated POAG patients compared with normals. Baskaran et a ${ }^{6}$ followed International Society for Geographical and Epidemiological Ophthalmology (ISGEO) classification for angle-closure disease. ${ }^{7}$ The combined PACG and primary angle-closure (PAC) group had more than twice the risk (odds ratio, 2.38) of having IOP fluctuation of more than $3 \mathrm{~mm} \mathrm{Hg}$ compared with the combined primary angle-closure suspect (PACS) and normal group. Although the subjects with PACG had the highest diurnal IOP fluctuation in both the above studies, the effect of the anti-glaucoma medications on the diurnal IOP fluctuation had not been studied in either of them. Moreover, Baskaran et $a l^{6}$ used a noncontact tonometer and not the gold standard Goldmann applanation tonometer (GAT) in their study. The treated POAG 
patients in previous studies on IOP fluctuation either do not truly represent treated POAG glaucoma as a whole or have issues in data collection. ${ }^{8-10}$ Baskaran et al ${ }^{6}$ enrolled study subjects after LPI but before commencement of any medical or surgical treatment. Current consensus recommends LPI in selected PACS eyes. ${ }^{11}$ Nevertheless, fluctuation of IOP in untreated PACS eyes is yet unreported.

The aim of this study was to evaluate IOP fluctuation during office hours and its predictive factors in untreated PACS, post-iridotomy PAC eyes with or without IOPlowering medications as appropriate, and treated PACG and POAG eyes.

\section{Materials and methods}

One hundred and eighteen subjects aged 40 years or older and consecutively attended the glaucoma clinic at a tertiary eye care hospital were enrolled in this study. All participants underwent gonioscopy using an indentation gonioscope. Nature of the angle was established in dim illumination taking care that the slit beam did not fall upon the pupil. The subjects with iridotrabecular apposition in whom the posterior trabecular meshwork was not visible for $180^{\circ}$ or more (modified from ISGEO definition ${ }^{7}$ ), without raised IOP, PAS, or optic nerve head changes consistent with glaucomatous optic neuropathy, were classified as PACS. We defined PAC as an eye with PACS and PAS and/or elevated IOP without glaucomatous damage of the optic disc; and PACG as an eye with PACS with evidence of glaucoma as defined by the ISGEO. ${ }^{7}$ We defined PAS in number of clock hour(s) with irido-trabecular apposition not broken by indentation gonioscopy. Subjects with IOP $>21 \mathrm{~mm} \mathrm{Hg}$ at the time of diagnosis, open drainage angles, and having optic disc changes consistent with glaucoma and corresponding visual field defects on automated static perimetry were termed POAG. The exclusion criteria were monocular status, previous intraocular surgery in either eye, history of acute angleclosure attack, presence of any corneal pathology, nanophthalmos, satisfying any criteria for doing LPI in PACS, advanced glaucoma with vertical cup-to-disc ratio (VCDR) $\geq 0.9$ : 1 with biarcuate glaucomatous field defects and macular involvement, pseudoexfoliation, pigment dispersion, other secondary glaucomas, and failure to apply scheduled dose of ocular hypotensive medication in the morning or the previous night, if any with respect to the day of office-hour IOP measurement. The criteria for doing LPI in PACS eyes were presence of PAC or PACG in the fellow eye, need for repeated dilated examinations, poor access to regular ophthalmic care, and confirmed family history of PACG. ${ }^{11}$
All subjects underwent a comprehensive ophthalmic examination and diagnostic procedures. Ocular biometry included keratometry (Bausch and Lomb Keratometer, Rochester, NY, USA); central anterior chamber depth (ACD), lens thickness (LT) and axial length (AXL, Alcon Ultrasonic Biometer, Ocuscan, Alcon Laboratories, TX, USA). The vertical corneal diameter and pupil diameter were measured using a centimeter scale. The diameter of pupil was studied in dim-room illumination. Central corneal thickness (CCT) was measured by an ultrasound pachymeter (Alcon Ultrasonic Biometer, Alcon, TX, USA). Peripheral ACD was graded as per the modified van Herick technique. ${ }^{12}$ A single trained observer (SS) performed four-mirror Posner gonioprism (Mentor, $\mathrm{OH}$, USA) assisted gonioscopy, adjudicated by an experienced glaucoma specialist (MB). The observer was masked to the diagnosis. The number of clock hours of PAS was specifically noted. Subjects with PAC and PACG underwent LPI before pupillary dilatation and at least 2 weeks prior to office-hour IOP recording. Following iridotomy, the subjects were asked to instill Prednisolone Acetate eye drops (Allergan, Bangalore, India) QID only for a week. Stereo-biomicroscopic disc evaluation was done with $+90 \mathrm{D}$ lens to record the VCDR along with neuroretinal rim changes. The eyes were categorized into three groups based on VCDR; which is $\leq 0.3,0.4-0.6$, and $\geq 0.7$. Humphrey visual field (HVF) 24-2 SITA Standard program (model 750, Carl Zeiss Meditec, Dublin, CA, USA) was performed in subjects with PAC, PACG, and POAG.

IOP readings were obtained hourly from 0800 to 1700 hours on a separate day using GAT. The ocular surface was anaesthetized with $0.5 \%$ Proparacaine eye drops (Sunways, Mumbai, India). IOP was always measured first in the right eye. A single observer (SS) performed all tonometry measurements using the same Goldmann applanation instrument. We checked the applanation instrument for calibration error(s) as per the manufacturer's instructions every morning prior to starting patient measurements. IOP reading at each hour was the mean of three consecutive IOP readings. Subjects were instructed to avoid drinking $\geq 500 \mathrm{ml}$ of any liquid $2 \mathrm{~h}$ before or during office-hour IOP recording. ${ }^{13}$ The subjects who were on TID medications received their medication as per schedule while undergoing the diurnal testing. The primary outcome measure was IOP fluctuation defined as the SD of office-hour IOP readings. The secondary outcome measure was IOP fluctuation defined as the range of IOP (peak minus trough) readings during office hours.

Statistical analysis was performed using SPSS 15.0 for Windows (SPSS Inc., Chicago, IL, USA). One eligible eye of each subject was considered for the analysis. If both the eyes were eligible and belonged to the same 
diagnostic criteria, one eye was chosen after randomization (MS Excel 2003, New York, NY, USA). The demographic and clinical data as well as peak, trough, range, and SD of IOP during office hours were compared between the groups. Continuous variables between groups were compared using parametric (oneway analysis of variance) and non-parametric analysis (Kruskal-Wallis test), and categorical variables were compared using the $\chi^{2}$-test for proportions or Fisher's exact test, as appropriate. Post hoc analysis between individual groups was performed by Tukey's or MannWhitney $U$-test for parametric or non-parametric data, respectively. A stepwise linear regression analysis was performed to assess the risk factors for IOP fluctuation among the angle-closure disease and POAG groups using age, gender, number of anti-glaucoma medications, mean keratometry, corneal diameter, CCT, pupil diameter, number of clock hours of PAS, ACD, LT, AXL, and VCDR as predictor variables. $P<0.05$ was considered statistically significant.

We certify that all applicable institutional and governmental regulations concerning the ethical use of human volunteers were followed during this research.

\section{Results}

One hundred and eighteen (30 PACS, 30 PAC (16 medically treated), 28 PACG, and 30 POAG) subjects were enrolled in this study. One patient with PACS had essential blepharospasm and was excluded from the analysis. No PACS subject did satisfy any criteria for LPI. All PACS subjects were untreated. All PAC and PACG subjects had LPI at least 2 weeks prior to office-hour IOP recording. Subjects with PAC, PACG, and POAG were on treatment with anti-glaucoma medications at the discretion of the treating ophthalmologist.

Demographic and clinical data are summarized in Table 1. IOP recruitment, corneal diameter, ACD, AXL, and mean (MD) and pattern SD (PSD) on HVF did differ significantly between the groups. Post hoc Mann-Whitney $U$-test did show significant difference in IOP at recruitment between PAC and all other groups (PACS vs PAC, $P<0.01$; PAC vs PACG, $P<0.01$; and PAC vs POAG, $P<0.01)$. Corneal diameter did differ only between PACS and POAG groups $(P<0.01)$. ACD differed significantly between all subtypes of angleclosure disease and POAG group (PACS vs POAG, $P<0.01$; PAC vs POAG, $P<0.01$; PACG vs POAG, $P<0.01)$ and also between PACS and PAC groups $(P=0.01)$. Similarly, AXL differed significantly between all subtypes of angle-closure disease and POAG group (PACS vs POAG, $P<0.01$; PAC vs POAG, $P<0.01$; PACG vs POAG, $P<0.01)$ and also between PACS and PAC

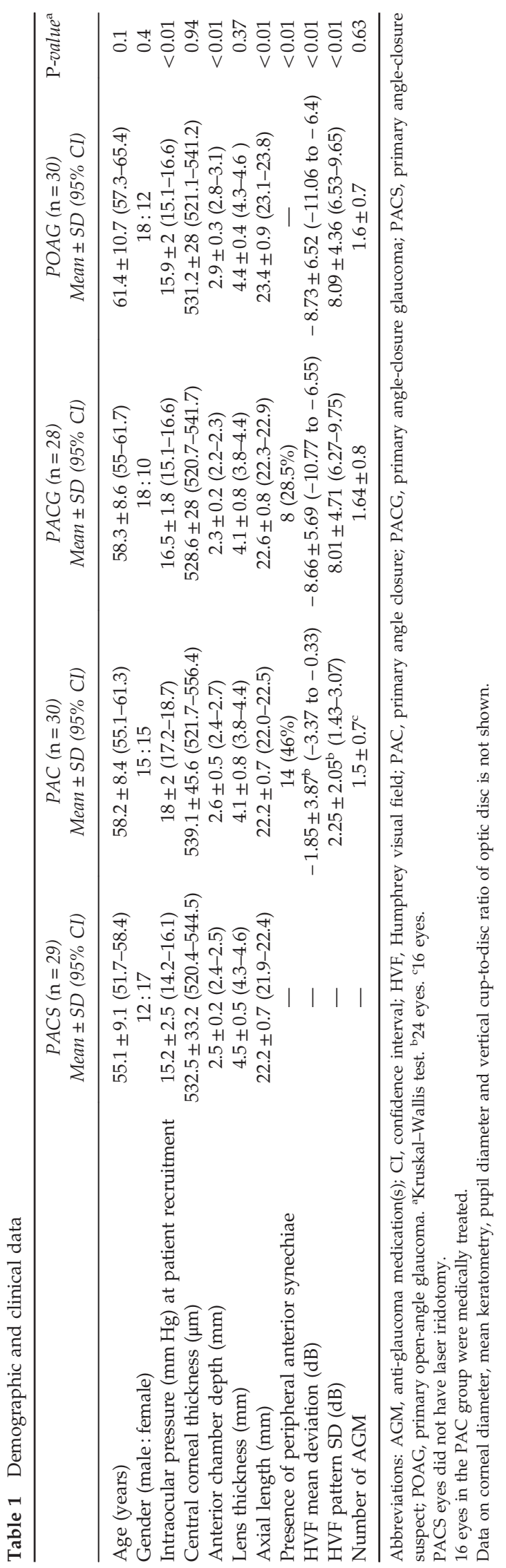


Table 2 Office-hour IOP

\begin{tabular}{|c|c|c|c|c|}
\hline Category & PACS, $\mathrm{n}=29$ & $P A C, \mathrm{n}=30$ & $P A C G, \mathrm{n}=28$ & $P O A G, \mathrm{n}=30$ \\
\hline $\begin{array}{l}\text { Peak office-hour IOP }{ }^{\text {a }}(P<0.01 \text {; } \\
\text { One-way ANOVA) }\end{array}$ & $17.1 \pm 2.28(16.23-17.96)$ & $20 \pm 3(18.92-21.07)$ & $19.29 \pm 3.12(18.07-20.5)$ & $17.17 \pm 3.79(15.81-18.52)$ \\
\hline $\begin{array}{l}\text { Trough office-hour } \operatorname{IOP}^{\mathrm{a}}(P<0.01 \text {; } \\
\text { One-way ANOVA) }\end{array}$ & $13.48 \pm 2.04(12.7-14.25)$ & $15.5 \pm 2.51(14.6-16.39)$ & $14.68 \pm 2.69(13.63-15.72)$ & $12.7 \pm 3.06(11.6-13.79)$ \\
\hline $\begin{array}{l}\text { SD of office-hour IOP measurements } \\
(P=0.01 ; \text { Kruskal-Wallis Test })\end{array}$ & $1.17(1.01-1.33)$ & $1.39(1.20-1.59)$ & $1.39(1.15-1.62)$ & 1.35 (1.17-1.53) \\
\hline $\begin{array}{l}\text { Range of IOP fluctuation during } \\
\text { office hours (mean } \pm S D ; P=0.09 \text {; } \\
\text { Kruskal-Wallis test) }\end{array}$ & $3.62 \pm 1.47$ & $4.5 \pm 1.5$ & $4.64 \pm 1.92$ & $4.47 \pm 1.69$ \\
\hline
\end{tabular}

Abbreviations: ANOVA, analysis of variance; $\mathrm{CI}$, confidence interval; IOP, intraocular pressure; PAC, primary angle closure; PACG, primary angle-closure glaucoma; PACS, primary angle-closure suspect; POAG, primary open-angle glaucoma. ${ }^{a} \mathrm{Mean} \pm \mathrm{SD}(95 \% \mathrm{CI})$.

groups $(P=0.03)$. Optic disc cupping was significantly different between the groups $\left(P<0.01 ; \chi^{2}\right.$-test $)$. Seventeen (58\%) PACS eyes belonged to VCDR category 1, 23 (76\%) PAC eyes belonged to VCDR category 2, whereas 20 (71\%) PACG and 26 (86\%) POAG eyes belonged to VCDR category 3. MD and PSD on HVF differed significantly between PAC and PACG $(P<0.01)$ and PAC and POAG groups $(P<0.01)$, respectively.

Table 2 shows office-hour IOP data. Peak IOP differed between PACS and PAC groups $(P=0.01), P A C$ and POAG groups $(P<0.01)$, and PACG and POAG groups $(P=0.02)$. Similarly, trough IOP differed significantly between PACS and PAC groups $(P<0.01)$, PACS and PACG groups $(P=0.04)$, and PAC and POAG groups $(P<0.01)$. The SD of office-hour IOP was significantly different between the groups $(P=0.01$; Kruskal-Wallis Test). Post hoc pair wise Mann-Whitney $U$-tests showed significantly different SD of office-hour IOP fluctuation only between PACS and PACG $(P<0.01)$. However, the range of IOP fluctuation during office hours did not significantly differ between the groups $(P=0.09$; KruskalWallis Test).

We observed peak IOP at 0900 hours in the PACS group (Figure 1a), at 1100 hours in the PAC group (Figure 1b), and around noon (at 1200 hours in the PACG group and at 1300 hours in the POAG group) in the glaucomatous groups (Figure 1c and d). We did not observe a statistically significant difference in the number of anti-glaucoma medications in the treatment groups $(P=0.63)$.

No eye with PACS or POAG had any PAS. Sixteen (53.3\%) eyes with PAC and 20 (71.4\%) eyes with PACG did not have PAS. Up to 3 clock hours of PAS were present in 13 (43.3\%) eyes with PAC and 4 (14.2\%) eyes with PACG. No eye had > 270 degree synechial angle closure. The pre-iridotomy extent of gonioscopic synechial angle closure is categorized in Table 3.

The factors that were positively correlated with SD of office-hour IOP were extent of clock hours of PAS
(Pearson's correlation coefficient $(r)=0.29, P<0.01)$, $\operatorname{VCDR}(r=0.18, P=0.04)$ and LT $(r=0.2, P=0.02)$.

A stepwise linear regression model identified the presence of PAS, thickness of the lens, large VCDR and PAC category as significant predictive factors associated with office-hour IOP fluctuation defined as SD of IOP (Table 4).

\section{Discussion}

We report IOP fluctuation during office hours in untreated PACS and treated PAC, PACG, and POAG. Office-hour IOP fluctuation in untreated PACS was significantly lower than that in the treated PACG and was at least comparable to that in treated PAC and POAG. The presence of PAS and thickness of the lens were the main predictive factors for IOP fluctuation during office hours.

PACS is defined by ISGEO as an eye in which $\geq 270^{\circ}$ appositional contact between the peripheral iris and posterior trabecular meshwork is considered possible with no structural or functional optic nerve damage. ${ }^{7}$ The propensity of appositional angle closure in PACS to cause IOP fluctuation is not completely explored. Sihota et $a l^{5}$ found comparable range of diurnal IOP fluctuation between chronic PACG $(7.69 \pm 3.03 \mathrm{~mm} \mathrm{Hg})$ and POAG $(8.31 \pm 2.58 \mathrm{~mm} \mathrm{Hg})$ eyes. However, the PACG eyes had undergone LPI and were medically untreated, and other subtypes of angle-closure disease were not studied.

Baskaran et $a l^{6}$ investigated IOP fluctuation during office hours in various forms of angle-closure disease. In their study, office-hour IOP fluctuation was significantly higher in PAC $(4.5 \pm 2.3 \mathrm{~mm} \mathrm{Hg})$ and PACG subjects $(5.4 \pm 2.4 \mathrm{~mm} \mathrm{Hg})$ compared with PACS subjects $(3.7 \pm 1.2 \mathrm{~mm} \mathrm{Hg})$ and normal controls $(3.8 \pm 1.1 \mathrm{~mm} \mathrm{Hg})$. The PAC and PACG groups showed higher mean IOP, peak IOP, and IOP fluctuation compared with PACS and normal groups. However, all angle-closure subjects (including those with PACS) were enrolled in the study 
a

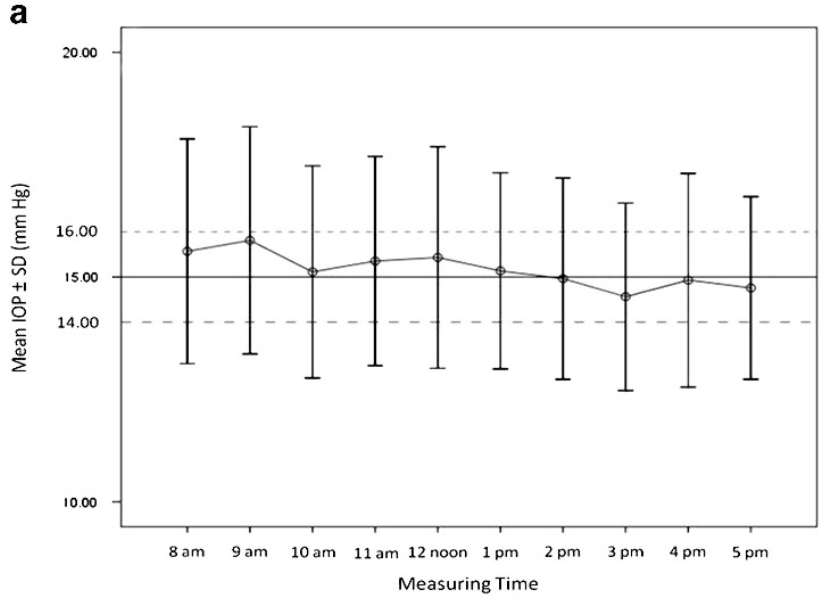

C

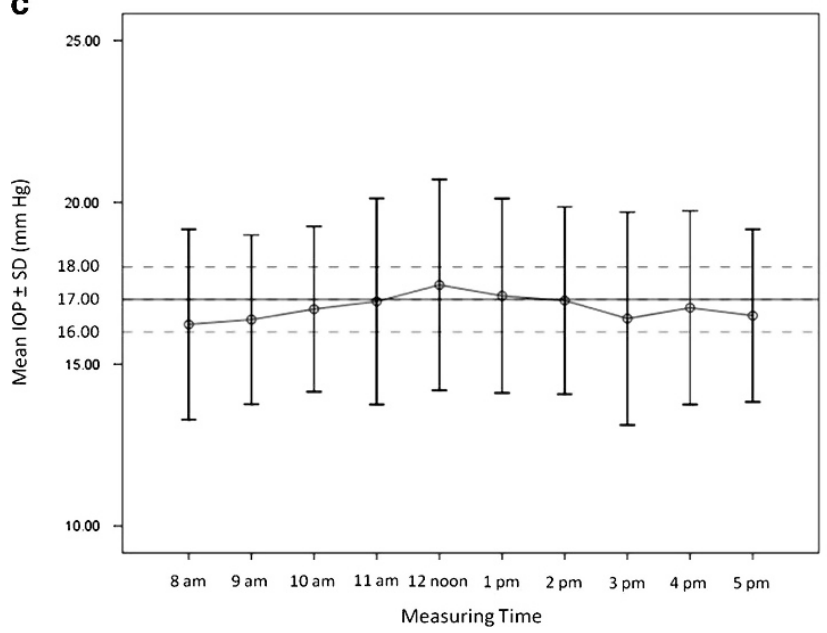

b

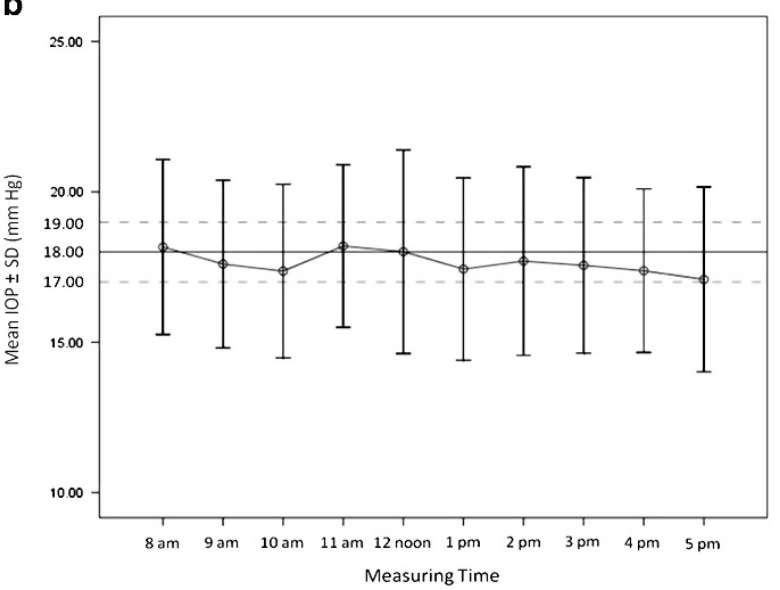

d

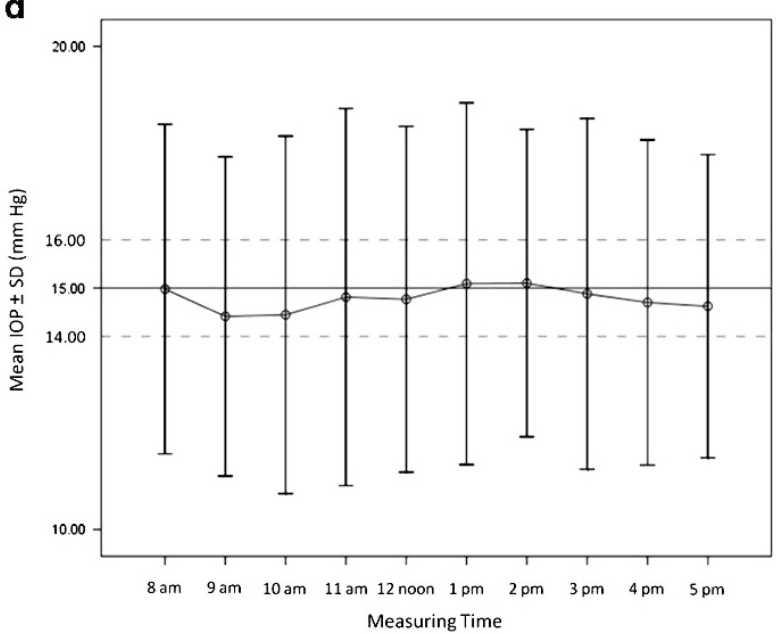

Figure 1 Intraocular pressure fluctuation during office hours in primary angle-closure suspect eyes (a), primary angle-closure eyes (b), primary angle-closure glaucoma eyes (c), and primary open-angle glaucoma eyes (d).

Table 3 Pre-iridotomy extent of synechial angle closure

\begin{tabular}{|c|c|c|c|c|c|}
\hline & $\operatorname{PACS}(\mathrm{n}=29)$ & $P A C(\mathrm{n}=30)$ & $P A C G(\mathrm{n}=28)$ & $P O A G(\mathrm{n}=30)$ & P-value ${ }^{\mathrm{a}}$ \\
\hline No PAS & $29(100 \%)$ & $16(53.3 \%)$ & $20(71.4 \%)$ & $30(100 \%)$ & $<0.01$ \\
\hline Up to 3 clock hours of PAS & 0 & $13(43.3 \%)$ & $4(14.2 \%)$ & 0 & \\
\hline 3 to 6 clock hours of PAS & 0 & $1(3.3 \%)$ & $2(7 \%)$ & 0 & \\
\hline 6 to 9 clock hours of PAS & 0 & 0 & $2(7 \%)$ & 0 & \\
\hline$>9$ clock hours of PAS & 0 & 0 & 0 & 0 & \\
\hline
\end{tabular}

Abbreviations: PAC, primary angle closure; PACG, primary angle-closure glaucoma; POAG, primary open-angle glaucoma; PACS, primary angle-closure suspect; PAS, Peripheral anterior synechiae. ${ }^{a}$ Significance testing by Fisher's exact test.

Table 4 Predictive factors associated with office-hour IOP fluctuation

\begin{tabular}{lccrr}
\hline Variables & Un-standardized coefficient $(B)$ & Standard error of $B$ & Standardized coefficient & P-value \\
\hline PAS (clock hours) & 0.10 & 0.03 & 0.25 & $<0.01$ \\
Lens thickness $(\mathrm{mm})$ & 0.17 & 0.05 & 0.25 & $<0.01$ \\
Large VCDR $(\geq 0.7)$ & 0.13 & 0.04 & 0.27 & $<0.01$ \\
PAC & 0.22 & 0.11 & 0.19 & 0.04 \\
\hline
\end{tabular}

Abbreviations: PAC, primary angle closure; PAS, peripheral anterior synechiae; VCDR, vertical cup-to-disc ratio.

PAC in comparison with PACS (PACS category was not coded). 
after LPI but before commencement of any medical or surgical treatment. ${ }^{6}$ In contrast, angle-closure suspects in our study had not undergone iridotomy. This is relevant since laser iridotomy to relieve the pupillary block is not recommended in all eyes with PACS. ${ }^{11}$ In addition, the subjects with PAC, PACG, as well as POAG were under anti-glaucoma medications as appropriate.

The IOP fluctuation during office hours in POAG eyes in our study was $4.47 \pm 1.69 \mathrm{~mm} \mathrm{Hg}$. The peak and trough mean IOPs in the POAG group were 17.17 \pm 3.79 and $12.7 \pm 3.06 \mathrm{~mm} \mathrm{Hg}$, respectively. These results compare well with earlier studies on treated POAG. ${ }^{8-10}$ However, these studies ${ }^{8,9}$ except the one by Jonas et al ${ }^{10}$ have reported IOP fluctuation during office hours in patients with POAG whose visual fields deteriorated despite acceptable office IOP levels or cases when a suspicious optic disc is discovered with normal office IOP. These patient groups may not truly represent treated POAG glaucoma as a whole. Jonas et al ${ }^{10}$ reported diurnal IOP profiles in open-angle and normal tension glaucoma. The range of circadian IOP fluctuation in 46 eyes under treatment for POAG was $5.74 \pm 3.44 \mathrm{~mm} \mathrm{Hg}$. However, the IOP measurements were performed by different observers using multiple Goldmann tonometers.

Baskaran et $a l^{6}$ discovered peak IOP in the morning hours between 0800 and 0900 hours in all subtypes of angle-closure disease and control eyes. Similarly, we found IOP peak at 0900 hours in the untreated PACS group. The around noon IOP peak in our POAG group was comparable to one study, ${ }^{8}$ but was in contrast to another, ${ }^{10}$ which reported IOP peak in early morning in treated POAG group. In the latter study, most of the patients were receiving topical $\beta$-blockers twice daily and the early morning IOP readings were obtained just before the morning dose of medication. This might have influenced the diurnal IOP profile. These findings may have implications for timing of tonometry.

The extent of PAS can be a surrogate marker for a compromised trabecular meshwork outflow resulting in diurnal IOP fluctuation. The result may suggest that the extent of PAS can be utilized as a clinical marker to make treatment decisions in angle-closure disease.

To our knowledge, biometric ocular measurements were not studied earlier as independent risk factors for diurnal IOP fluctuation in glaucoma. In our study, thickness of the lens was the only biometric factor associated with significant IOP fluctuation during office hours. Use of ocular hypotensive medications could have prevented our finding any association between biometric parameters and office-hour IOP fluctuation. Moreover, ocular biometric parameters themselves have been shown to have a significant diurnal fluctuation. ${ }^{14}$
Our study identified larger VCDR as a risk factor for IOP fluctuation during office hours. Similarly Baskaran et $a l^{6}$ found another marker of severity of glaucoma, which is degree of visual field loss to be significantly associated with office-hour IOP fluctuation in patients with angle-closure disease. However, our study cannot establish cause and effect relationship between larger VCDR and IOP fluctuation. Larger VCDR might be the effect and not the cause of larger IOP fluctuation. Moreover, VCDR is dependent upon the size of the optic disc and, therefore, is not steady information.

Our study has few limitations. First, we did not have a control group of normal eyes. LPI and IOP-lowering medications change fluctuation of IOP. This might have negated any intergroup difference with regard to our secondary outcome measure, which is range of IOP fluctuation during office hours. However, current consensus recommends LPI only in selected PACS eyes and, therefore, we were interested in studying IOP fluctuation in untreated PACS and comparing it with that in other varieties of primary glaucoma under treatment as appropriate. We did not exclude data from any subject on the basis of failure of the subject to apply scheduled dose of ocular hypotensive medication with respect to the day of office-hour IOP measurement; however, we did not formerly assess compliance of the study subjects with their medications. Various other factors may affect diurnal IOP recording, such as blood pressure fluctuation, squeezing of eyelids, ocular pulse amplitude, and a tight collar. ${ }^{15}$ We did not study these factors. The magnitude of nocturnal IOP fluctuation might be larger than the diurnal fluctuation seen in this study particularly in angle-closure suspects without LPIs; however, we did not study nocturnal IOP variation. We did not attempt to classify plateau iris and study it as a risk factor for diurnal IOP fluctuation. In addition, we did not do HVF in PACS eyes for logistic reason, and therefore, did not use HVF data in linear regression.

In summary, this study has shown for the first time that the office-hour IOP fluctuation was significantly lower in untreated PACS than that in treated PACG and was at least comparable to that in treated PAC and POAG. Peak diurnal IOP was observed in the morning in the untreated subjects and in early afternoon in the treated subjects, warranting differing IOP monitoring protocols and appropriate treatment modifications. The presence of PAS and increased LT were found to be the significant risk factors for office-hour IOP fluctuation. A larger VCDR as a surrogate marker for the severity of the disease was also associated with higher IOP fluctuation during office hours. 


\section{Summary}

What was known before

- Fluctuation of IOP, both during and beyond office hours is an independent risk factor for the progression of openangle glaucoma.

\section{What this study adds}

- Office-hour intraocular pressure (IOP) fluctuation in untreated primary angle-closure glaucoma suspects (PACS): Office-hour IOP fluctuation was significantly lower in untreated PACS than that in treated primary angle-closure glaucoma and was at least comparable to that in treated primary angle closure and primary openangle glaucoma.

- Risk factors for office-hour IOP fluctuation: The presence of peripheral anterior synechiae and increased lens thickness were found to be the significant risk factors for office-hour IOP fluctuation in glaucoma.

\section{Conflict of interest}

The authors declare no conflict of interest.

\section{Acknowledgements}

This study was funded by Vision Research Foundation, Chennai, India.

\section{References}

1 Chiseliţă D, Moţoc I, Danielescu C. Daily and nightly fluctuation of intraocular pressure and blood pressure in glaucoma and non-glaucoma patients. Oftalmologia 2008; 52: 119-125 Romanian.

2 Tajunisah I, Reddy SC, Fathilah J. Diurnal variation of intraocular pressure in suspected glaucoma patients and their outcome. Graefes Arch Clin Exp Ophthalmol 2007; 245: 1851-1857.

3 Musch DC, Gillespie BW, Niziol LM, Lichter PR, Varma RCIGTS Study Group. Intraocular pressure control and long-term visual field loss in the Collaborative Initial Glaucoma Treatment Study. Ophthalmology 2011; 118: 1766-1773.
4 Asrani S, Zeimer R, Wilensky J, Gieser D, Vitale S, Lindenmuth $\mathrm{K}$. Large diurnal fluctuations in intraocular pressure are an independent risk factor in patients with glaucoma. J Glaucoma 2000; 9: 134-142.

5 Sihota R, Saxena R, Gogoi M, Sood A, Gulati V, Pandey RM. A comparison of the circadian rhythm of intraocular pressure in primary chronic angle closure glaucoma, primary open angle glaucoma and normal eyes. Indian J Ophthalmol 2005; 53: 243-247.

6 Baskaran M, Kumar RS, Govindasamy CV, Htoon HM, Wong CY, Perera SA et al. Diurnal intraocular pressure fluctuation and associated risk factors in eyes with angle closure. Ophthalmology 2009; 116: 2300-2304.

7 Foster PJ, Buhrmann R, Quigley HA, Johnson GJ. The definition and classification of glaucoma in prevalence surveys. Br J Ophthalmol 2002; 86: 238-242.

8 Collaer N, Zeyen T, Caprioli J. Sequential office pressure measurements in the management of glaucoma. J Glaucoma 2005; 14: 196-200.

9 David R, Zangwill L, Briscoe D, Dagan M, Yagev R, Yassur Y. Diurnal intraocular pressure variations: an analysis of 690 diurnal curves. Br J Ophthalmol 1992; 76: 280-283.

10 Jonas JB, Budde WM, Stroux A, Oberacher-Velten IM, Juenemann AG. Circadian intraocular pressure profiles in chronic open angle glaucomas. J Ophthalmic Vis Res 2010; 5: 92-100.

11 South East Asia Glaucoma Interest Group. Asia Pacific Glaucoma Guidelines. 2008:31. Available from http://www. icoph.org/dynamic/attachments/resources/asia-pacific_ glaucoma_guidelines.pdf. Accessed on November 2015.

12 Foster PJ, Devereux JG, Alsbirk PH, Lee PS, Uranchimeg D, Machin $\mathrm{D}$ et al. Detection of gonioscopically occludable angles and primary angle closure glaucoma by estimation of limbal chamber depth in Asians: modified grading scheme. Br J Ophthalmol 2000; 84: 186-192.

13 Susanna R Jr, Medeiros FA, Vessani RM, Giampani J Jr, Borges AS, Jordão ML. Intraocular pressure fluctuations in response to water drinking provocative test in patients using Latanoprost Versus Unoprostone. J Ocul Pharmacol Ther 2004; 20: 401-410.

14 Chakraborty R, Read SA, Collins MJ. Diurnal variations in axial length, choroidal thickness, intraocular pressure, and ocular biometrics. Invest Ophthalmol Vis Sci 2011; 52: 5121-5129.

15 Chihara E. Assessment of true intraocular pressure: the gap between theory and practical data. Surv Ophthalmol 2008; 53: 203-218. 